\title{
CARACTERIZANDO RELACIONES ENTRE LA RESOLUCIÓN DE PROBLEMAS Y LA DEMOSTRACIÓN EN ÁLGEBRA ABSTRACTA
} CHARACTERIZING RELATIONSHIPS BETWEEN PROBLEM SOLVING AND PROOF IN ABSTRACT ALGEBRA

\author{
Mauricio Penagos ${ }^{1}$ \\ Rafael Julio Sánchez Lamoneda ${ }^{2}$ \\ Luis Fernando Mariño ${ }^{3}$
}

\section{RESUMEN}

La investigación desarrollada con 12 estudiantes de licenciatura en matemáticas tuvo como fin dar respuesta a la pregunta científica ¿cómo son las relaciones entre la resolución de problemas y la demostración en algebra abstracta, manifestada por profesores de matemáticas en formación? Para la recolección de información

$1 \quad$ Licenciado en Matemáticas y Física Universidad Surcolombiana, Docente de Tiempo Completo Universidad Surcolombiana, Maestro en Ciencias en Física, Doctor (C) en Educación Matemática, e-mail: mauriciopenagos@usco. edu.co. Orcid: https://orcid.org/0000-0002-4524-2914

2 Docente e investigador Doctorado en Educación Matemática de la Universidad Antonio Nariño (Colombia). Máster en Matemáticas (Instituto Pedagógico de Caracas, Venezuela). PhD en Matemáticas (Brandeis University, USA). Correo. lamonedar@uan.edu.co. Orcid: 0000-00027012-4928

3 Doctor en Educación Matemática (Universidad Antonio Nariño, Colombia). Magister en Educación Matemática. Licenciado en Matemáticas y Computación. Profesor Departamento de Matemáticas y Estadística de la Universidad Francisco de Paula Santander (Cúcuta). Correo. fernandoml@ufps.edu.co. Orcid: https://orcid. org/0000-0002-3438-69632. se implementaron seis actividades didácticas, dos encuestas semiestructuradas, como complemento para triangular la información se tomó la opinión a docentes de álgebra abstracta y finalmente se aplicó a los estudiantes una encuesta de satisfacción. Para el análisis de datos se tuvo en cuenta la teoría de la Dualidad, Necesidad y Razonamiento Repetido de Harel y Sowder, así como la propuesta de Schoenfeld sobre resolución de problemas. Los hallazgos muestran que tanto al resolver problemas como al demostrar teoremas se encuentran patrones iguales en el abordaje, el uso de representaciones, los procesos de solución, justificación y verificación. Los resultados muestran que en el contexto del algebra abstracta no se encuentran diferencias en los procesos de solución de problemas y demostración de teoremas, por el contrario, son procesos que se complementan uno al otro, además hay incidencia de factores psicológicos, 
didácticos y creencias sobre la matemática que repercuten en el desarrollo de ambos procesos.

\section{PALABRAS CLAVE:}

Resolución de Problemas, Demostración Matemática, Álgebra Abstracta, Dualidad, Necesidad y Razonamiento Repetido

\section{ABSTRACT}

The research developed with 12 undergraduate students in mathematics was aimed at answering the scientific question: how are the relationships between problem solving and demonstration in abstract algebra, as expressed by mathematics teachers in training? For the collection of information, six didactic activities were implemented, two semi-structured surveys, as a complement to triangulate the information, the opinion of abstract algebra teachers was taken and finally a satisfaction survey was applied to students. For data analysis, Harel and Sowder's theory of Duality, Necessity and Repeated Reasoning was taken into account, as well as Schoenfeld's proposal on problem solving. The findings show that when solving problems and proving theorems, the same patterns are found in the approach, the use of representations, the processes of solution, justification and verification. The results show that in the context of abstract algebra there are no differences in the processes of problem solving and theorem proving; on the contrary, they are processes that complement each other, and there are also psychological and didactic factors and beliefs about mathematics that have an impact on the development of both processes.

\section{KEYWORDS:}

Problem Solving, Mathematical Demonstration, Abstract Algebra, Duality, Necessity and Repeated Reasoning.

\section{INTRODUCCIÓN}

La resolución de problemas es un motivador para producir conocimiento matemático, mientras que la demostración de teoremas está íntimamente ligada a procesos deductivos y argumentativos, permitiendo afianzar conceptos y avanzar hacia la abstracción. Rodríguez et al (2019) consideran que, en el aprendizaje de la matemática, el estudiante se asemeja a un matemático, ya que trabaja con problemas y realiza la misma actividad: explora, conjetura, analiza, etc.

El carácter abstracto y generalizador del álgebra permea otras ramas de la matemática y otras ciencias, sin embargo, muchas de sus bondades son opacadas por sus formas de enseñanza que repercuten negativamente en el aprendizaje de los estudiantes. Dubinsky y otros (1994) afirman que, en ocasiones los estudiantes consideran que es una asignatura compleja con alto grado de abstracción matemática, exigiendo al estudiante memorizar axiomas, definiciones y teoremas necesarios al momento de realizar demostraciones y resolver problemas. Este carácter axiomático ocasiona en algunos casos se pierda el gusto por esta materia.

En la mayoría de los programas de formación de profesores de matemáticas se ofrece al menos un curso de estructuras algebraicas. Findell (2001) considera que en estos cursos los estudiantes pueden extraer características comunes de sistemas matemáticos estudiados en otros cursos y pueden fortalecer sus habilidades de pensamiento algebraico. Le permite también al futuro docente mejorar la comprensión de los contenidos matemáticos, aprender a analizar y escribir demostraciones, además de constituir un punto de referencia para que los docentes en formación alcancen niveles más altos de abstracción (Edwards \& Brenton, 1999).

En las matemáticas, la heurística se refiere a las estrategias para guiar o descubrir. Polya 
(1945) afirma que se relaciona con los métodos y las reglas de descubrimiento e invención. Schoenfeld (1985) por su parte, considera que la heurística guía la resolución exitosa de problemas porque le permiten al individuo comprender mejor la situación y avanzar hacia la solución. Según McDonald, citado por Hughes (1974) es un modelo de procesamiento de información que produce procedimientos efectivos para proceder, conjeturar, resolver problemas y hacer demostraciones.

Polya (1945) propone un método de cuatro fases para resolver problemas: entender el problema, elaborar un plan, ejecutar el plan y mirar hacia atrás (revisar el trabajo). Schoenfeld (1985), propone una metodología de resolución de problemas consistente en cuatro etapas: análisis, exploración, ejecución y comprobación, a la vez tiene en cuenta otros factores importantes como los recursos, las heurísticas, el control y las creencias sobre las matemáticas.

En lo que respecta a la demostración matemática algunos investigadores la consideran como una secuencia de acciones físicas (escribir o dibujar) y mentales (recordar); cada acción es una respuesta a una situación interna propia del individuo y que relaciona varias áreas del conocimiento (Knapp, 2003). Harel \& Sowder $(1998,2007)$ proponen tres tipos de esquemas de demostración de teoremas: externos, empíricos y analíticos, los cuales representan distintas etapas del desarrollo matemático del individuo.

Los esquemas empíricos pueden ser de tipo perceptivo o inductivo, son aquellos en los cuales el estudiante utiliza ejemplos para demostrar resultados; la fuente es el razonamiento inductivo proveniente de sus percepciones sensoriales, conocimientos previos, lo observado en ejemplos, sustituir en fórmulas u observar patrones con los que puede validar casos particulares. Mientras que, en los esquemas de demostración externa, el individuo se convence así mismo y trata de persuadir a otros con argumentos de procedencia externa. Entre tanto, los esquemas analíticos ocurren cuando la validación de una conjetura se obtiene a partir de procesos lógico-deductivos y pueden ser transformacionales o axiomáticos.

Muchos investigadores de educación matemática destacan la relación existente entre resolver problemas y demostrar teoremas. Para hacer demostraciones matemáticas se requiere tener conocimiento sólido, destrezas para resolver problemas, capacidad de direccionar los saberes, reformular ideas, redacción, síntesis y la adopción de conductas que tienen los expertos matemáticos. Knapp (2003) considera que los estudiantes aprenden a demostrar cuando están inmersos en las matemáticas y que en los planes estudio son necesarios los cursos que promuevan la definición, la conjetura, la resolución de problemas y la demostración.

Para Savic (2015) la demostración matemática y que al revisar la literatura sobre resolución de problemas se pueden describir aspectos de la demostración, ya que esta última es una consecuencia de la resolución de problemas. Este autor plantea que en estas tareas ocurren tres fases: planificar, ejecutar y verificar, además de la existencia de una fase llamada orientación incluida en la planificación.

Weber (2005) por su parte, plantea que en la resolución de problemas se enfatiza en los procesos de pensamiento para avanzar en el desarrollo de la situación y que la demostración se enfatiza más en evaluar la solidez de un resultado; considera que hay aspectos comunes en ambas actividades, pues ambas tienen que ver con la producción de argumentación matemática.

Mientras que, Selden y Selden (2013) afirman que al hacer demostraciones deben tenerse en 
cuenta dos aspectos: la parte retórica formal o marco de la prueba y la parte centrada en el problema. La primera depende de la interpretación, los preconceptos y la lógica para entender la formulación. La parte centrada en el problema tiene que ver con la teoría de resolución de problemas en el sentido de Schoenfeld.

Tanto la resolución de problemas como la demostración en algebra abstracta han sido abordados por una gran variedad de educadores matemáticos, profesores e investigadores de forma independiente. Ante este panorama e intentando explorar las relaciones entre la demostración y resolución de problemas el trabajo tuvo como propósito dar respuesta a la pregunta de investigación: ¿cómo son las relaciones entre la resolución de problemas y la demostración en algebra abstracta, manifestada por profesores de matemáticas en formación?

\section{METODOLOGÍA}

Para responder la pregunta orientadora se optó por un enfoque cualitativo, puesto que el propósito del estudio estuvo centrado en analizar, dar sentido y descubrir relaciones en las manifestaciones escritas de los participantes cuando resuelven problemas $\mathrm{y}$ hacen demostraciones de algebra abstracta. En el diseño metodológico se tuvo en cuenta el enfoque de investigación-acción, pues lo asumen personas que desarrollan una actividad conjunta en su beneficio, mediante la reflexión, interactúan la teoría y la práctica y no hay distinción entre lo que se investiga, quién investiga y el proceso (Colmenares \& Piñero, 2008).

\section{PARTICIPANTES}

La constituyó 12 estudiantes que tomaron un curso de Estructuras Algebraicas durante el segundo semestre académico de 2020 del programa de Licenciatura en Matemáticas de la Universidad Surcolombiana de Neiva, institución de carácter oficial y modalidad presencial. Los participantes se ubicaban en el rango de edad entre 19 y 24 años, se trataba de 5 mujeres y 7 hombres. Debido al aislamiento social a causa de la pandemia ocasionada por el virus SARSCoV-2, el desarrollo de las actividades se hizo mediante encuentros virtuales sincrónicos y asincrónicos utilizando la plataforma Meet de Google.

\section{FUENTES DE DATOS}

Durante el semestre académico de 16 semanas de duración se aplicaron a los estudiantes dos encuestas semiestructuradas, una en la tercera semana de clases y la otra en la novena semana. Con el fin era triangular la información las encuestas también se aplicaron a docentes de álgebra abstracta y a expertos. Se diseñaron, validaron e implementaron un sistema de seis actividades didácticas, en las que se pidió a los estudiantes, sin decirles si es lo uno o lo otro, que demuestren teoremas y que resuelvan problemas de álgebra abstracta.

Un objetivo transversal de las actividades fue analizar la argumentación escrita y verbal (a través de entrevistas) de los estudiantes al resolver problemas y demostrar teoremas sobre algunos de los temas de álgebra abstracta estudiados en el curso: métodos de demostración, operaciones binarias, grupos y subgrupos, grupos cíclicos, clases laterales y homomorfismo de grupos. En cada actividad se especificaba el tema, los objetivos, los materiales necesarios contaba con un encabezamiento, el tiempo aproximado de duración y al final se formulaban los problemas teoremas que debían resolver.

\section{ANÁLISIS DE DATOS}

De las encuestas semiestructuradas. La primera encuesta se aplicó a los estudiantes en la tercera semana del semestre para indagar su percepción sobre la resolución de problemas 
y demostración de teoremas. Se le pregunta también si consideran que hay diferencias significativas en el esfuerzo mental cuando desarrollan una y otra tarea. A continuación, se resumen los resultados encontrados:

En cuanto a las dificultades para resolver problemas y demostrar teoremas, los estudiantes consideran que residen en la interpretación de los enunciados, el manejo lógico-deductivo $y$ que piensan que se resuelven aplicando fórmulas o desarrollando algoritmos. Los docentes afirman que a los estudiantes se les dificulta utilizar definiciones y resultados matemáticos, generar esquemas mentales y modelos de representación, que no implementan estrategias, desconocen los métodos de demostración y les falta dedicación. Según los expertos a los estudiantes se les dificulta argumentar tanto en las demostraciones como en los procesos de resolución de problemas; que ven las matemáticas como un conjunto de técnicas $y$ fórmulas, prefieren lo cuantitativo y lo práctico y que se presenta un rechazo automático a situaciones que requieran demostrar o argumentar.

En relación con la forma de razonar (los procesos mentales involucrados) cuando resuelven problemas $y$ demuestran teoremas de álgebra abstracta, 8 de los 12 estudiantes consideraban que existen diferencias. Uno de los cinco docentes participantes también cree lo mismo y argumenta:

los objetos algebraicos son construcciones mentales abstractas y al razonar con ellos en una demostración, la mayoría de las veces no se tiene soportes fácticos lo que conlleva a esfuerzos mentales más exigentes". Uno de los expertos $(20 \%)$ considera también que existen diferencias y afirma que "los esquemas de pensamiento tienen sustento sobre conceptos, juicios y razonamientos. Cuando se resuelven problemas, el alumno está en un ambiente más familiar, mientras que al demostrar teoremas influyen otros procesos como las creencias y concepciones, que se pide probar un resultado que es verdadero y debe construirse la cadena de argumentos para la justificación.

Todos los participantes consideran que en la demostración matemática y la resolución problemas de álgebra abstracta no ocurren procesos exclusivamente cognitivos, cuando entienden lo que se les pregunta sienten satisfacción, autoestima, confianza y deseo de continuar. Ocurre lo contrario cuando no comprenden el enunciado, o cuando llegan a un punto donde ya no pueden continuar, esto los lleva a una sensación de frustración e impotencia. Los docentes y expertos por su parte consideran que en ocasiones los estudiantes manifiestan alegría, otras veces nerviosismo, inseguridad, ansiedad o duda incluso de lo que saben o entienden; en ocasiones frustración y desespero.

En cuanto a las representaciones que más utilizan los estudiantes al resolver problemas y demostrar teoremas de álgebra abstracta, los participantes coinciden en las siguientes: dibujos, gráficos, tablas o esquemas que 
les permitan simplificar y reducir la dificultad, ejemplos y contraejemplos antes de iniciar un proceso riguroso de solución o demostración, analizar casos particulares para tener claridad al inicio, durante o al final del proceso.

\section{ANÁLISIS DEL SISTEMA DE ACTIVIDADES}

Primera Actividad, métodos de demostración. Se realizó en la segunda semana de clases a manera de pilotaje y el objetivo fue analizar los preconceptos con que llegan los estudiantes al curso de álgebra y su desempeño en la solución de problemas y demostración de teoremas. La Figura 1, muestra el trabajo del participante codificado como E7-AMD1 (E7 significa estudiante 7 y AMD1) luego resolver la tarea: Demuestre que los números reales y son raíces de la ecuación cuadrática

$x^{2}+p x+q=0$ si y sólo sí $a+b=-p$ y $a b=q$

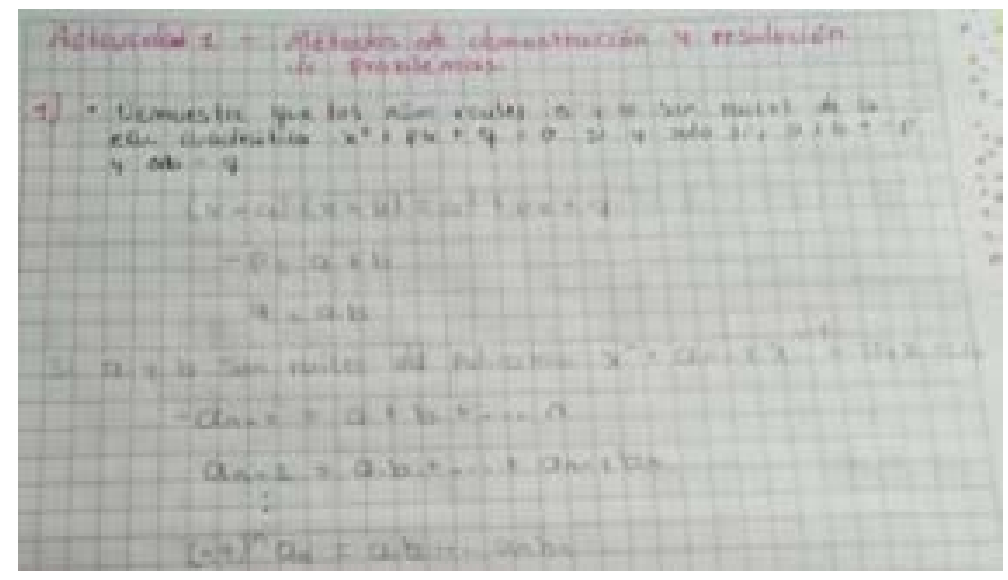

Figura 1. Manifestaciones escritas E7-AMD1

Sin ofrecer detalles el estudiante procura demostrar la condición de suficiencia de la proposición. La segunda parte no conduce a una conclusión coherente que pruebe la condición de necesidad. Al indagar al estudiante sobre su respuesta, manifiesta que:

Escribe lo que entiende. Que recibió apoyo de sus compañeros $y$ de lo que lo entendió en lo que investigó, porque había encontrado un problema con un enunciado parecido. Que lo importante es entregar la actividad resuelta para no sacar mala nota.

Esta solución corresponde el esquema de convicción externa, propuesto por Harel y Sowder (1988), según la cual el individuo se convence por lo observado en libros o por lo que les asegura otra persona al que creen sus argumentos. También se asemeja al esquema simbólico ya que el estudiante manipula símbolos matemáticos, sin comprender bien su significado.

En la Figura 2, se observa el trabajo escrito del estudiante codificado como E9-AMD2, al realizar la tarea, donde se pide que demuestre o refute la siguiente proposición: sí que si es un entero positivo, entonces $n^{2}+n+41$, es un número primo. 


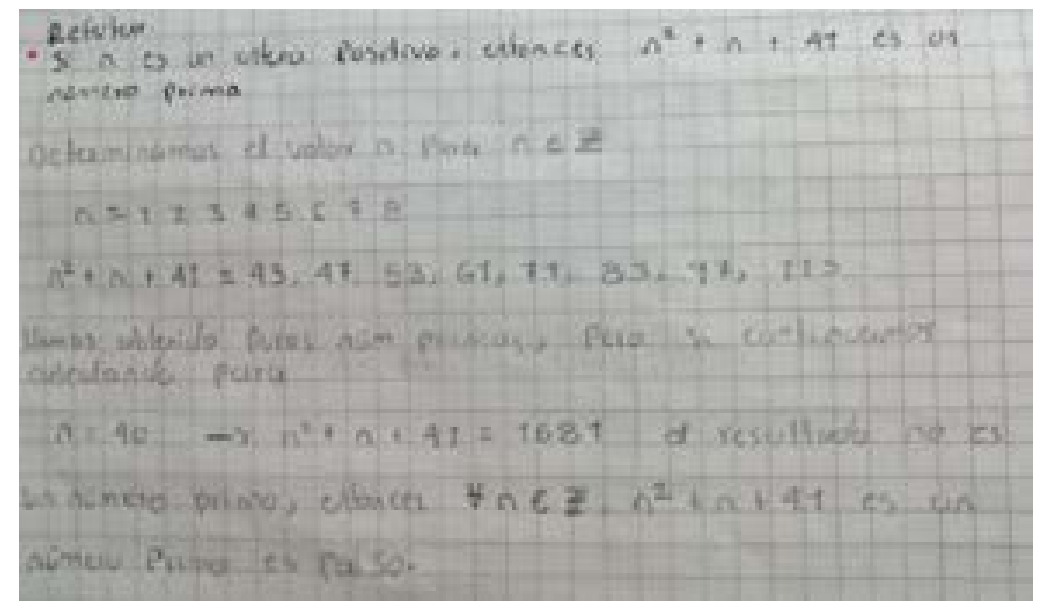

Figura 2. Manifestación escrita E9-AMD2

Al indagarle por la solución ofrecida, el estudiante manifiesta:

me cuesta trabajo este tipo de problemas porque en los cursos anteriores no se trabaja la resolución de problemas así y tampoco me ha tocado hacer demostraciones. El profesor explica la teoría, presenta sin demostrar algunos resultados importantes y siguen los ejercicios de práctica
Esta actuación está en concordancia con los esquemas de demostración empíricos propuestos por Harel \& Sowder (1988) que son argumentos inductivos, en este caso sustentados por sustituir en una fórmula matemática y en la observación de patrón que le permite generar ejemplos particulares.

En la Figura 3 se observa el trabajo del estudiante E10-AMD3, al realizar la tarea: Demuestre que los números reales $a$ y $b$, son raíces de ecuación cuadrática:

$$
x^{2}+p x+q=0 \Leftrightarrow a+b=-p \quad y \quad a b=q
$$

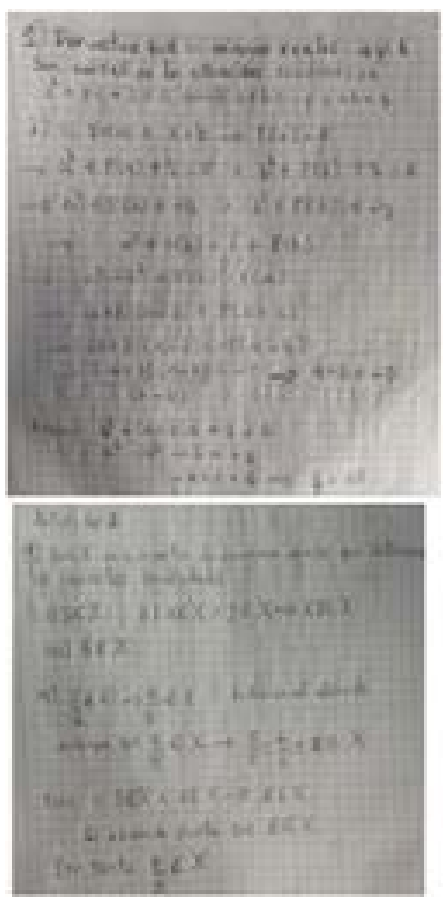

Figura 3.

Demostración E10-AMD3 
Se destaca su esfuerzo por escribir de manera coherente, aunque se observan algunas limitaciones; se entiende lo que quiere expresar y se percibe mejor dominio conceptual. Al indagar al estudiante sobre sus argumentos, explica lo escrito y es consciente de que los resultados matemáticos están soportados en definiciones, axiomas, propiedades $y$ otros resultados matemáticos y que para demostrar debe haber una articulación coherente entre estos.

El procedimiento encaja en los esquemas de demostración analíticos de Harel y Sowder (1998) porque utiliza la deducción lógica para validar la conjetura tanto del problema como de la proposición. La argumentación es transformacional - no axiomática- porque realiza un proceso deductivo que está orientado a objetivos definidos.

\section{Segunda y tercera actividad, grupos $y$ subgrupos.}

Se implementó en la octava semana. El objetivo era analizar la argumentación escrita y verbal de los estudiantes cuando demuestran teoremas y cuando resuelven problemas sobre grupos y subgrupos. La Figura 4, evidencia el trabajo del estudiante E10-AGSG1:
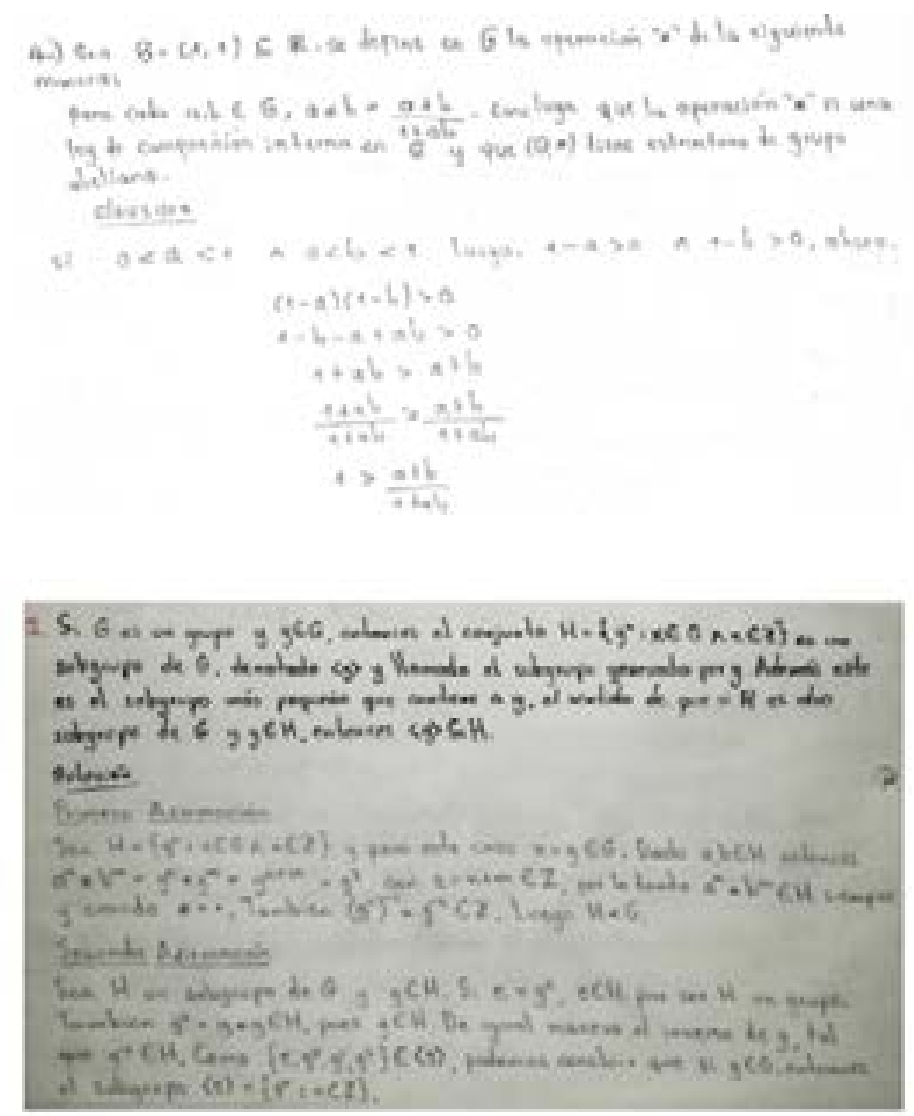

Figura 4. Manifestaciones E10-AGSG1

En la figura 4, al lado izquierdo se pide resolver un problema. En la solución expuesta se percibe que el estudiante tiene en cuenta axiomas de los números reales, un adecuado manejo de símbolos, un desarrollo ordenado, igualmente los pasos que sigue están relacionados y justifican el proceso; hay avances en la abstracción y la generalización. Los aspectos descritos anteriormente son también propios de la demostración de teoremas.

Al lado de derecho de la misma figura 4 se 
muestra la prueba que realizó otro estudiante a una proposición y allí se refleja, también la adecuada manipulación analítica de las variables y la aceptación y aplicación de definiciones, axiomas y resultados propios de la estructura de grupos; esto conduce según Radford (2006) al desarrollo del pensamiento algebraico.

Por otro lado, en la solución de ambas actividades hay vestigios de los esquemas axiomáticos propuestos por Harel y Sowder (1988): se observa que los estudiantes deducen los resultados como consecuencia lógica de teorías, axiomas y resultados que consideran válidos. En la entrevista, afirman que para resolver lo propuesto en las actividades (ya sea problema o teorema) deben razonar muy

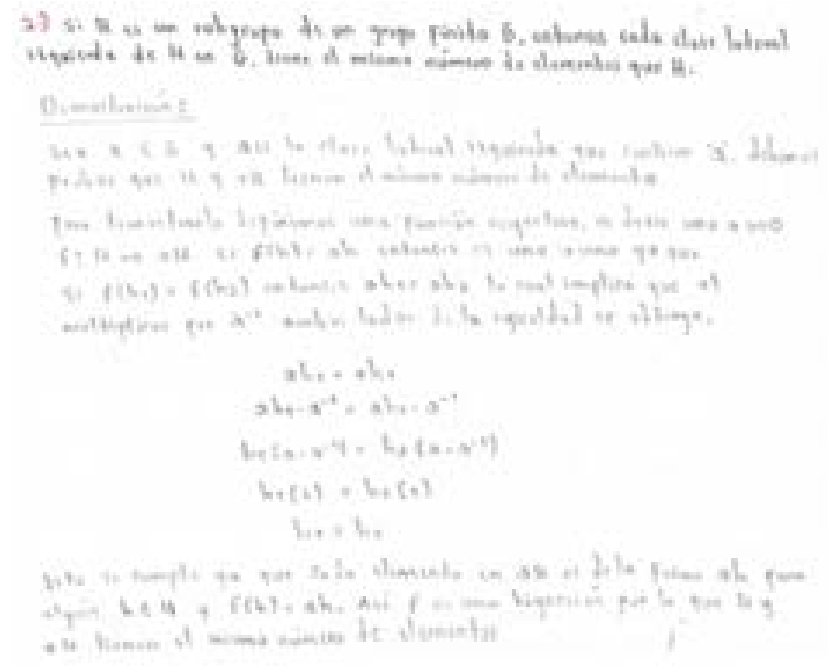

bien, conocer la teoría, ser organizados, aplicar estrategias adecuadas y que en uno y otro caso tuvieron momentos en que avanzaron, otros en que se pararon y otros en los que le tocó retroceder y que cuando tienen éxito en la solución de las actividades la interiorización del resultado les facilita resolver otros problemas y demostrar.

Cuarta actividad, clases laterales. Se propuso en la doceava semana de clases y el objetivo fue analizar la argumentación escrita y verbal (entrevistas) de los estudiantes al demostrar teoremas y resolver problemas sobre clases laterales. La Figura 5, muestra la solución de un problema y una demostración por parte del estudiante E7-CL1

Figura 5. Solucionando problemas y demostrando E7-CL1

Se destaca que ya sea que se trate de un teorema o de un problema, en el contexto del álgebra abstracta para conseguir una solución válida, se necesita conocer la teoría para justificar los pasos, ambas actividades requieren de niveles de abstracción, análisis, implementación de estrategias y procesos de generalización similares.
La figura 5 también pone en evidencia los procesos lógico-deductivos y la manipulación analítica de los objetos matemáticos. Se ratifican lo planteado por De Villiers (1990) quien considera que los estudiantes aprenden a demostrar si comprenden la funcionalidad de la demostración y que esta permite a los individuos ir más allá del rol de verificación y tiene un 
efecto positivo en el compromiso que asume en la comprensión del resultado.

Quinta y sexta actividad, homomorfismos de grupos. El objetivo se centró en analizar la argumentación escrita y verbal (mediante entrevistas) de los estudiantes cuando resuelven problemas y demuestran teoremas sobre homomorfismo de grupos y fue implementada en la semana 15. La Figura 6, muestra el trabajo del participante E10-AHG1.
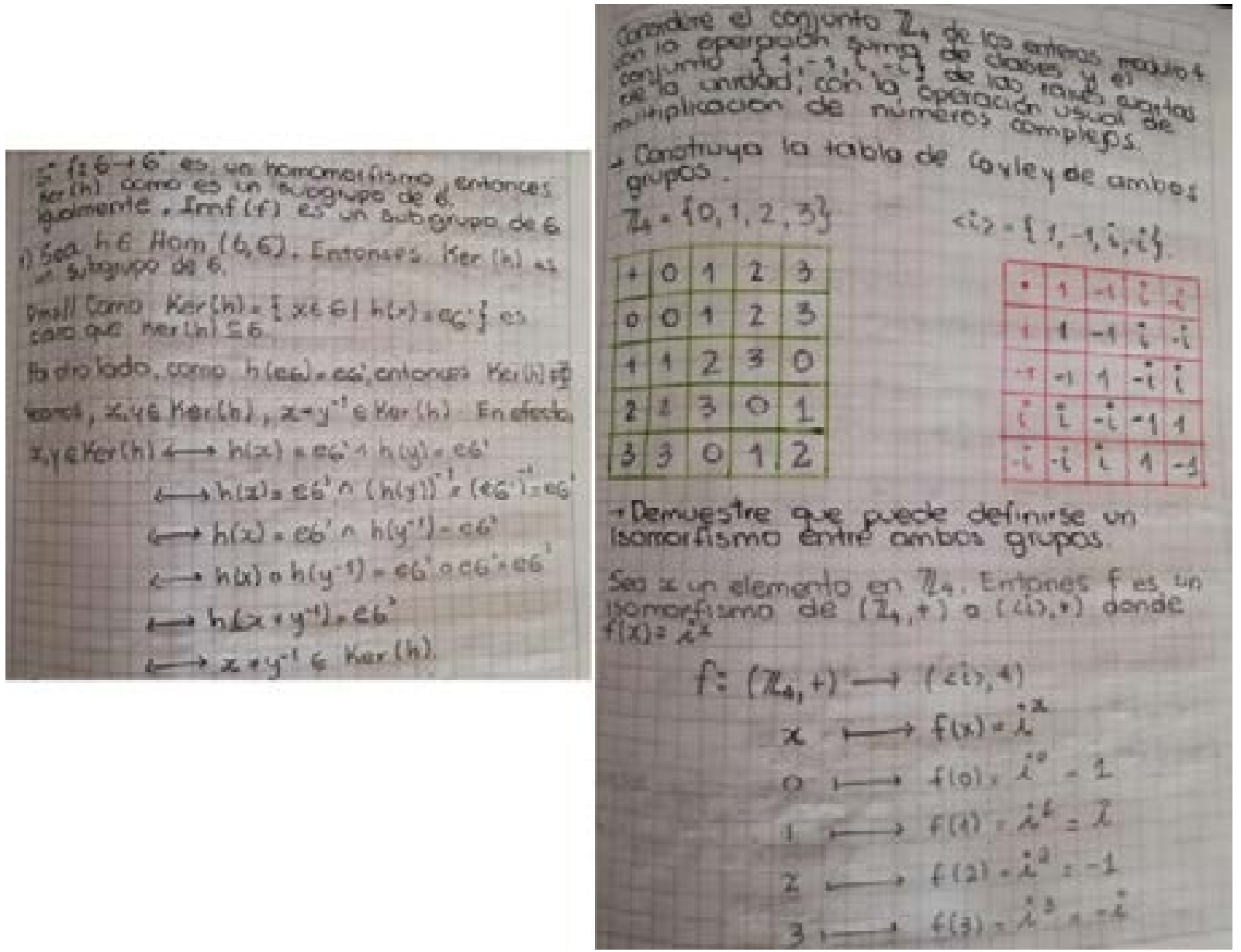

Figura 6. Trabajando con homomorfismos E10-AHG1

Entre los principales hallazgos, en la figura 6, a la izquierda se propone la demostración de un teorema y a la derecha un problema. En ambas situaciones se observan esquemas de demostración analíticos, propios de un nivel significativo de generalización. Se validan los resultados de Knapp (2003), que para demostrar se requiere tener buen conocimiento de la teoría, destrezas para resolver problemas, capacidad de direccionar los conocimientos y reformular ideas, redacción, síntesis matemática y el desarrollo de conductas que tienen los expertos en hacer demostraciones.

Sin importar que el enunciado aparezca la palabra demuestre, se observa en los participantes la implementación adecuada de estrategias heurísticas, progresos significativos en la capacidad de resolver problemas, una notable manipulación de los objetos matemáticos que les permite trascender de lo concreto a lo abstracto. Las entrevistas permitieron concluir que, en este punto de la investigación es 
irrelevante que en la actividad se pida demostrar un teorema o resolver un problema, pues la mayoría de los estudiantes coincide en que el proceso de solución guarda estrecha relación y que ambas actividades están vinculadas y que en cuanto al esfuerzo mental no encuentran diferencias significativas.

Se validan también los resultados encontrados por Knapp (2003) en relación con los pasos seguidos por los participantes en el desarrollo de la actividad didáctica: intuición, ensayo, error, especulación, conjetura y demostración la cual es una secuencia natural (no lineal) para desarrollar el pensamiento matemático.

\section{RESULTADOS Y DISCUSIÓN}

Para responder a la pregunta de investigación ¿Qué tipo de relaciones manifiestan los profesores de matemáticas en formación cuando resuelven problemas $y$ hacen demostraciones de algebra abstracta? se puso en ejecución la metodología antes expuesta junto a la implementación de las encuestas y las actividades didácticas. A continuación, se muestran los resultados:

A medida que avanza la investigación y lograr que los estudiantes estuvieran inmersos en la resolución de más problemas y la demostración de teoremas de álgebra abstracta, finalmente cerca del $75 \%$ de los 12 participantes estuvieron de acuerdo en que, en el contexto del álgebra abstracta para demostrar teoremas y resolver problemas se requiere un esfuerzo mental equivalente. Por otro lado, el $16.7 \%$ de los estudiantes le es indiferente que existan o no diferencias en la forma de razonar y el $8.3 \%$ considera aún que los procesos mentales son diferentes. Posiblemente esto se debe a que las actividades didácticas propuestas escapan a situaciones puramente concretas, circunstancias numéricas, a elementos del contexto o a manejos lineales y algorítmicos. Por lo tanto, ya sea problema o teorema se requiere un alto grado de análisis y razonamiento.

Tanto al resolver problemas como al demostrar teoremas se encuentran patrones iguales en el abordaje, el uso de representaciones, los procesos de solución, justificación y verificación. El análisis de datos permite afirmar que la demostración matemática es una actividad de resolución de problemas y quienes son exitosos resolviendo problemas utilizan técnicas similares cuando hacen demostraciones. Confirmando así que, desde el contexto del álgebra abstracta, la resolución de problemas y la demostración de teoremas se superponen, ya que esta última es un subconjunto de la primera, una consecuencia y que la literatura sobre resolución de problemas permite describir aspectos de la demostración matemática como lo propone Savic (2015).

En la medida en que los estudiantes se enfrentan con verdaderos problemas de álgebra abstracta y no con simples ejercicios, su percepción sobre las relaciones (semejanzas o diferencias) en la forma en que deben aproximarse y resolverlos en comparación con la demostración de teoremas cambia, a tal punto que manifiestan que el procedimiento es el mismo. El esquema de la Figura 7, representa esta afirmación. 


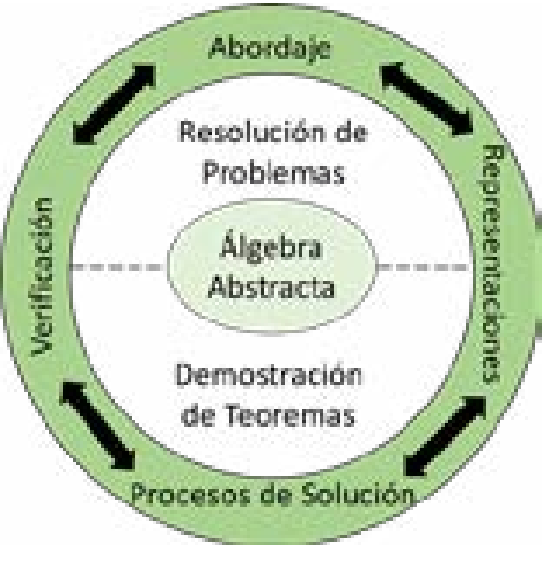

Figura 7. Similitudes entre resolver problemas y demostrar

Por otro lado, el $83.3 \%$ de los estudiantes del grupo considera que, tanto en la resolución de problemas como en la demostración de teoremas de álgebra abstracta, además de lo cognitivo influyen factores psicológicos, didácticos y creencias sobre la matemática. Al restante $16.7 \%$ le resulta indiferente.

Las entrevistas permitieron concluir que los mencionados factores pueden ocurrir más de uno a la vez, que están relacionados y afectan la dimensión cognitiva, y en consecuencia inciden en el desempeño de los estudiantes, el cual está enmarcado en triunfos, dificultades, alegría y frustración, etc. Se reconoce que las creencias sobre la matemática y la dimensión afectiva del individuo son prioritarias. Los autores resumen lo anterior en el esquema de la Figura 8.

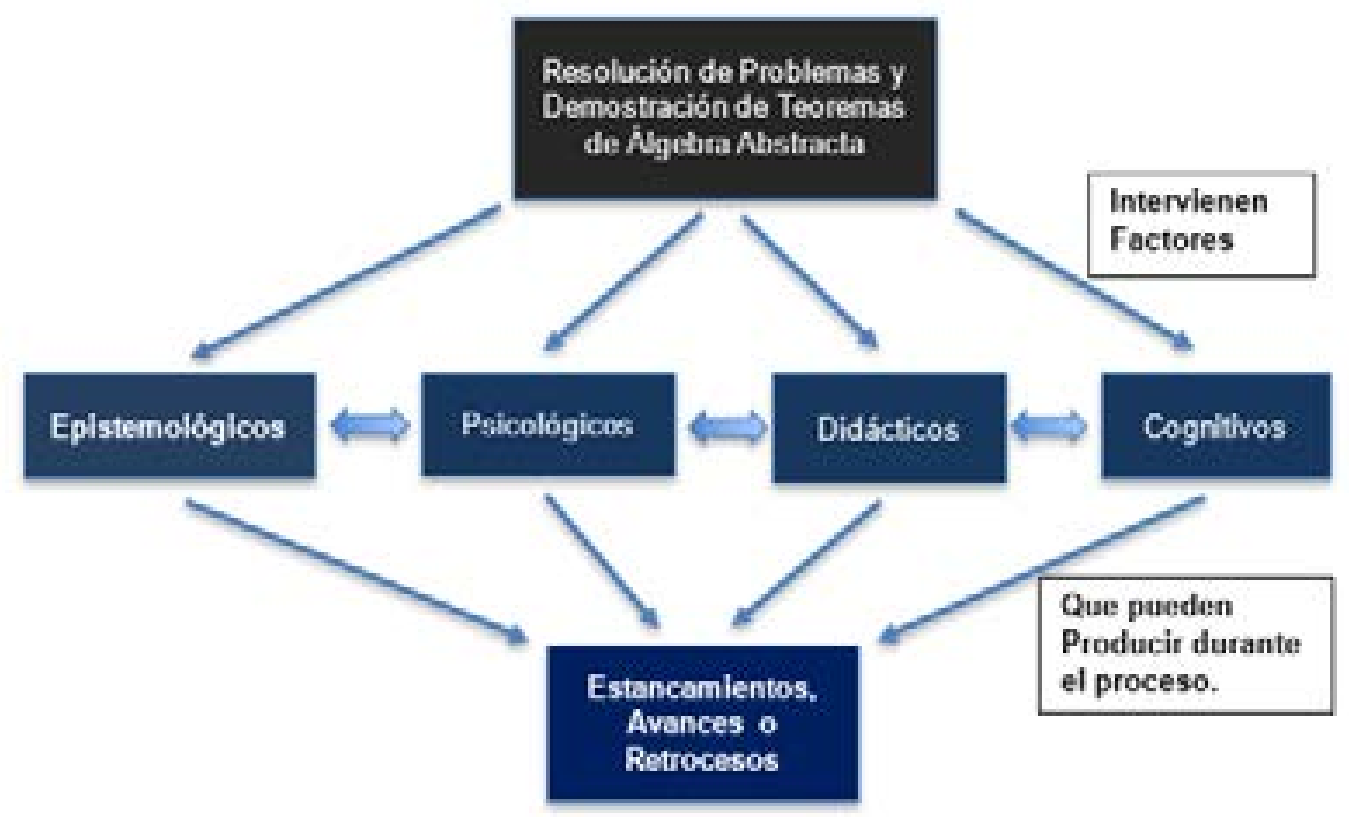

Figura 8. Factores que inciden en la solución de problemas y la demostración

Los teoremas, en particular los de álgebra abstracta, se caracterizan por su valor teórico, el nivel de generalización y la importancia para fundamentar otros resultados. En este campo, una demostración como resolver un problema implican una interacción cognitiva entre la formulación y el individuo, quien desde sus preconceptos e intuición interpreta, articula sus saberes, busca estrategias y en un proceso no lineal y menos inmediato busca la solución. El éxito le permite alcanzar un resultado que lo convenza a él y persuadir a otros tal como lo propone Harel y Sowder (1998). 
En cuanto a la validez y credibilidad de los resultados encontrados, los autores ratifican su validez en atención a que son el resultado de las respuestas ofrecidas por los participantes a las encuestas, entrevistas y al sistema de actividades implementadas, todas direccionadas a encontrar relaciones (diferencias 0 semejanzas) en la forma de razonar cuando los docentes en formación se enfrentaban a la solución de problemas o a demostrar teoremas de álgebra abstracta. Es preciso aclarar que las situaciones seleccionadas y planteadas en las actividades no es la única, ya que obedecen exclusivamente a la decisión de los autores; en tal sentido otros investigadores pueden decidir actividades para teorizar de forma diferente en una investigación similar.

Finalmente, los autores destacan los avances logrados con los participantes y recomienda que cuando se motiva a los estudiantes a resolver problemas, se producen cambios positivos en su confianza, lo que influye positivamente en lo afectivo y en su desempeño, lo que favorece el desarrollo de su pensamiento matemático. Investigaciones similares, podrían implementarse en otras áreas de la matemática como la teoría de números y el análisis, por recomendar algunas, para indagar si pueden encontrarse relaciones entre resolver problemas y demostrar teoremas.

\section{REFERENCIAS BIBLIOGRÁFICAS}

Colmenares, A. y Piñero, M. (2008). La Investigación Acción. Una herramienta metodológica heurística para la comprensión y transformación de realidades y prácticas socioeducativas. Laurus, 14(27), 96-114.

De Villiers, M. D. (1990). The role and function of proof in mathematics. Pythagoras, 24, $17-24$
Dubinsky E. \& Otros. (1994). On Learning Fundamental Concepts in Group Theory. Recuperado de http://www.math.kent. edu/ edd/FundConGrpTh.pdf.

Edwards T. \& Brenton L. (1999). An Attempt to Foster Students' Construction of Knowledge during a Semester Course in Abstract Algebra. The College Mathematics Journal, Vol. 30, No. 2 (Mar., 1999), pp. 120-128. Recuperado de: https://www.jstor.org/ stable/2687722.

Findell, B. (2001). Learning and Understanding in Abstract Algebra (tesis doctoral). Universidad de New Hampshire, U.S.A.

Harel, G. \& Sowder, L. (1998). Student's Proof Schemes. Research on Collegiate Mathematics Education, Vol. III. En Dubinsky, E., Schoenfeld, A. \& Kaput, J. (Eds.) (234-283.). AMS.

Harel, G. (2007). The DNR System as a Conceptual Framework for Curriculum Development and Instruction. En Lesh, R., Kaput, J. \& Hamilton, E. (Eds.) (pp. 263-280). Foundations for the Future in Mathematics Education, Erlbaum.

Hughes, B. (1974). Heuristic Teaching in Mathematics. Educational Studies in Mathematics, 5(3), 291-299.

Knapp J. (2003). Learning to Prove in Order to Prove to Learn. Department of Mathematics, Arizona State University, Tempe, Arizona 85287. Recuperado de http://citeseerx.ist.psu.edu/viewdoc/dow nload?doi=10.1 1.489.9566\&rep=rep1\& type $=$ pdf

Polya, G. (1945). How to Solve It: A New Aspect of Mathematical Method. New Jersey, U.S.A: Anchor Books Edition. 
Radford, L. (2006). Algebraic Thinking and The Generalization of Patterns: A Semiotic Perspective. PME-NA 2006 Proceedings. Vol. 1-2.

Rodríguez, M. et al. (2019). Heurísticas en la resolución de problemas matemáticos 1a ed. Los Polvorines: Universidad Nacional de General Sarmiento, Argentina. 84 p. Recuperado de https:// ediciones.ungs.edu.ar/wp-content/ uploads/2020/04/9789876304405completo.pdf.

Savic, M (2015). On Similarities and Differences Between Proving and Problem Solving. Journal of Humanistic Mathematics, Volume 5, Issue 2. July 2015.

Schoenfeld, A. (1985). Mathematical Problem Solving. Florida, U.S.A: Academic Press Inc.

Selden, A. \& Selden, J. (2013). Proof and Problem Solving at University Level. The Mathematics. Enthusiast: Vol. 10: No. 1, Article 14. Available at: https:// scholarworks.umt.edu/tme/vol10/ iss $1 / 14$.

Weber K. (2005). Problem-solving, proving, and learning: The relationship between problem-solving processes and learning opportunities in the activity of proof construction. Journal of Mathematical Behavior 24 (2005) 351-360. 